\title{
Metformin protects high glucose-cultured cardiomyocytes from oxidative stress by promoting NDUFA13 expression and mitochondrial biogenesis via the AMPK signaling pathway
}

\author{
XIANG-DONG LIU ${ }^{1 *}$, YONG-GUANG $\mathrm{LI}^{2 *}$, GUANG-YU WANG ${ }^{2}$, YA-GUANG BI $^{2}$, YU ZHAO ${ }^{2}$, \\ MEI-LING YAN ${ }^{2}$, XUEBO LIU ${ }^{1}$, MENG WEI $^{2}$, LI-LI WAN ${ }^{3}$ and QING-YONG ZHANG ${ }^{4}$ \\ ${ }^{1}$ Department of Cardiology, Tongji Hospital, School of Medicine, Tongji University, Shanghai 200065; \\ ${ }^{2}$ Department of Cardiology; ${ }^{3}$ Division of Pharmacy, Shanghai Jiao Tong University Affiliated Sixth People's Hospital, \\ Shanghai 200233; ${ }^{4}$ Department of Cardiology, Shanghai Ninth People's Hospital, Shanghai Jiaotong University \\ School of Medicine, Shanghai 200011, P.R. China
}

Received December 2, 2019; Accepted June 1, 2020

DOI: $10.3892 / \mathrm{mmr} .2020 .11599$

\begin{abstract}
Tissue damage in diabetes is at least partly due to elevated reactive oxygen species production by the mitochondrial respiratory chain during hyperglycemia. Sustained hyperglycemia results in mitochondrial dysfunction and the abnormal expression of mitochondrial genes, such as NADH: Ubiquinone oxidoreductase subunit A13 (NDUFA13). Metformin, an AMP-activated protein kinase (AMPK) activator, protects cardiomyocytes from oxidative stress by improving mitochondrial function; however, the exact underlying mechanisms are not completely understood. The aim of the present study was to investigated the molecular changes and related regulatory mechanisms in the response of H9C2 cardiomyocytes to metformin under high glucose conditions. H9C2 cells were subjected to CCK-8 assay to assess cell viability. Reactive oxygen species generation was measured with DCFH-DA assay. Western blotting was used to analyze the expression levels of NDUFA13, AMPK, p-AMPK and GAPDH. Reverse transcription-quantitative PCR was used to evaluate the expression levels of mitochondrial genes and transcription factors. It was observed that metformin
\end{abstract}

Correspondence to: Professor Qing-Yong Zhang, Department of Cardiology, Shanghai Ninth People's Hospital, Shanghai Jiaotong University School of Medicine, 639 Zhizaoju Road, Shanghai 200011, P.R. China

E-mail: zhangqingyong6th@126.com

Professor Li-Li Wan, Division of Pharmacy, Shanghai Jiao Tong University Affiliated Sixth People's Hospital, 600 Yishan Road, Shanghai 200233, P.R. China

E-mail:wanlily3533@163.com

${ }^{*}$ Contributed equally

Key words: metformin, cardiomyocyte, mitochondrial biogenesis, reactive oxygen species protected H9C2 cardiomyocytes by suppressing high glucose (HG)-induced elevated oxidative stress. In addition, metformin stimulated mitochondrial biogenesis, as indicated by increased expression levels of mitochondrial genes (NDUFA1, NDUFA2, NDUFA13 and manganese superoxide dismutase) and mitochondrial biogenesis-related transcription factors [peroxisome proliferator-activated receptor-gamma coactivator-1 $\alpha$, nuclear respiratory factor (NRF)-1, and NRF-2] in the metformin + $\mathrm{HG}$ group compared with the HG group. Moreover, metformin promoted mitochondrial NDUFA13 protein expression via the AMPK signaling pathway, which was abolished by pretreatment with the AMPK inhibitor, Compound $\mathrm{C}$. The results suggested that metformin protected cardiomyocytes against HG-induced oxidative stress via a mechanism involving AMPK, NDUFA13 and mitochondrial biogenesis.

\section{Introduction}

Diabetic patients have an notably increased incidence of heart failure according to the Framingham study (1); however, the molecular mechanisms underlying the pathogenesis of diabetic cardiomyopathy are not completely understood. Among the hypothesized mechanisms, hyperglycemia-induced oxidative stress is recognized as a critical participant in the pathogenesis and progression of diabetes (2). Increased levels of oxidative stress lead to harmful modifications of macromolecules, including DNA, proteins and lipids (3), which can result in cardiomyocyte apoptosis, hypertrophy and fibrosis, ultimately leading to cardiac remodeling and dysfunction (3). Therefore, developing an effective treatment to suppress increased oxidative stress levels and subsequent cardiomyocyte injury in patients with diabetes is important.

Metformin is widely used as a first-line hypoglycemic drug (4). Previous studies identified cardioprotective effects of metformin beyond its antihyperglycemic effects (5-7). The United Kingdom Prospective Diabetes Study demonstrated that early and intensive metformin intervention could reduce the incidence of myocardial infarction and increase the survival rate in patients with type 2 diabetes (5). Metformin 
exerts cardioprotective effects partly by suppressing high glucose (HG)-induced excessive reactive oxygen species production and inflammatory responses (8); however, the exact underlying mechanisms are not completely understood.

AMP-activated protein kinase (AMPK), a key mediator of the downstream effects of metformin, can be activated by cytokines, hormones and oral hypoglycemic agents that are commonly used to treat type 2 diabetes (9). Activated AMPK promotes the production of ATP by regulating glucose and fatty acid metabolism (9), indicating that AMPK may serve as an innate survival mechanism for the heart. For example, AMPK activation during myocardial ischemia reduces the infarct size in the hearts of diabetic model rats (10). In addition, upregulation of AMPK signaling in the vasculature improves microvascular function in the hearts of diabetic model mice (11).

Based on its role in regulating cellular energy status, AMPK is a major regulator of mitochondrial function. Hydroxytyrosol, a natural antioxidant, reduces oxidative stress and improves mitochondrial function, presumably by activating AMPK signaling in the brain of $\mathrm{db} / \mathrm{db}$ mice (12). Therefore, it was hypothesized that AMPK may serve as a critical modulator of mitochondrial biogenesis and function, and enhance resistance to oxidative stress. However, the exact role and mechanisms underlying AMPK in the cardioprotective effects of metformin in response to oxidative stress are not completely understood.

Mitochondria are the primary energy generating organelles, but are also the main source of ROS (13). NADH: Ubiquinone oxidoreductase subunit A13 (NDUFA)13 is an indispensable assembly factor of complex I (14). The primary role of NDUFA13 in the antioxidant effect of resveratrol has been previously reported (15). Downregulated NDUFA13 expression increases mitochondrial ROS generation in $\mathrm{H} 9 \mathrm{C} 2$ cardiomyocytes (16), and sustained high glucose decreases NDUFA13 expression levels (15).

Based on the aforementioned studies, it was hypothesized that NDUFA13 may be associated with alleviating oxidative stress in diabetic cardiomyopathy, and metformin may prevent diabetic myocardial injury by suppressing hyperglycemia-induced ROS overproduction. The present study aimed to investigate whether the protective effects of metformin were mediated by promoting mitochondrial biogenesis and NDUFA13 expression. Additionally, the exact role of the AMPK signaling pathway in metformin-induced effects was investigated.

\section{Materials and methods}

Cell culture and treatments. $\mathrm{H} 9 \mathrm{C} 2$ cells (The Cell Bank of Type Culture Collection of the Chinese Academy of Sciences) were cultured in DMEM (Gibco; Thermo Fisher Scientific, Inc.) supplemented with $10 \%$ FBS (Gibco; Thermo Fisher Scientific, Inc.), $100 \mathrm{U} / \mathrm{ml}$ penicillin and $100 \mathrm{mg} / \mathrm{ml}$ streptomycin at $37^{\circ} \mathrm{C}$ in a humidified incubator with $5 \% \mathrm{CO}_{2}$ and $95 \%$ air.

H9C2 cells cultured to approximately $80 \%$ confluence were firstly incubated with various concentrations of glucose $(5.5,15,25,33.340$ and $50 \mathrm{mM})$ for $36 \mathrm{~h}$ at $37^{\circ} \mathrm{C}$. Cells treated with various concentrations of glucose and mannitol (Abcam) were then incubated for $12,24,36$ and $48 \mathrm{~h}$ at $37^{\circ} \mathrm{C}$. These included 5.5 and $33.3 \mathrm{mM}$ glucose and $25 \mathrm{mM}$ glucose plus $8.3 \mathrm{mM}$ mannitol. According to changes in cell viability, $5.5 \mathrm{mM}$ glucose was selected to mimic normal conditions and $33.3 \mathrm{mM}$ glucose was selected to mimic the diabetic condition. Subsequently, cells were treated with low concentrations of metformin ( 0.5 or $1 \mathrm{mM}$; Sigma-Aldrich; Merck $\mathrm{KGaA}$ ) for $36 \mathrm{~h}$ at $37^{\circ} \mathrm{C}$. For subsequent experiments, $1 \mathrm{mM}$ metformin was used, which was proven to be an effective in previous studies $(17,18)$. The cardiomyocytes were divided into the following experimental groups: i) Control, medium containing $5.5 \mathrm{mM}$ glucose; ii) HG, medium containing $33.3 \mathrm{mM}$ glucose; iii) mannitol, medium containing $25 \mathrm{mM}$ glucose $+8.3 \mathrm{mM}$ mannitol; iv) metformin $0.5 \mathrm{mM}$, medium containing $33.3 \mathrm{mM}$ glucose $+0.5 \mathrm{mM}$ metformin; v) Met 1 , medium containing $33.3 \mathrm{mM}$ glucose $+1 \mathrm{mM}$ metformin; and vi) Compound $\mathrm{C}$, medium containing $33.3 \mathrm{mM}$ glucose $+1 \mathrm{mM}$ metformin $+10 \mu \mathrm{M}$ Compound C (Sigma-Aldrich; Merck KGaA).

Cell Counting Kit-8 (CCK-8) assay for cell viability. Cell viability was assessed using a CCK-8 assay kit (Shanghai Yeasen Biotechnology Co., Ltd.) according to the manufacturer's protocol. $\mathrm{H} 9 \mathrm{C} 2$ cells were seeded $\left(5 \times 10^{4}\right.$ cells/well $)$ into 96-well plates. Subsequently, CCK-8 reagent was added to each well and incubated for $1 \mathrm{~h}$ at $37^{\circ} \mathrm{C}$. The absorbance of each well was measured at a wavelength of $450 \mathrm{~nm}$ using a microplate reader (BioTek Instruments, Inc.).

Lactate dehydrogenase $(L D H)$ release. Cell death was assessed using an LDH cytotoxicity assay kit (Shanghai Yeasen Biotechnology Co., Ltd.) according to the manufacturer's instructions. Following treatment for $36 \mathrm{~h}$, the cell medium was collected. The absorbance of each sample was measured at a wavelength of $450 \mathrm{~nm}$ using a microplate reader.

Measurement of ROS, malondialdehyde (MDA) and superoxide dismutase (SOD). Intracellular ROS levels were determined using a ROS assay kit (Shanghai Yeasen Biotechnology Co., Ltd.) according to the manufacturer's protocol. Dichlorofluorescein fluorescence was detected using an Olympus IX71 fluorescence microscope (Olympus Corporation; magnification, x200). MDA levels were assessed using an MDA assay kit (Beyotime Institute of Biotechnology) according to the manufacturer's instructions. The absorbance of each sample was measured at a wavelength of $535 \mathrm{~nm}$ using a microplate reader. SOD activity was measured using a total SOD assay kit (Shanghai Yeasen Biotechnology Co., Ltd.) according to the manufacturer's protocol. The absorbance of each sample was measured at a wavelength of $450 \mathrm{~nm}$ using a microplate reader.

Western blotting. The H9c2 cells were lysed with RIPA buffer (Beyotime Institute of Biotechnology) for $45 \mathrm{~min}$ at $4^{\circ} \mathrm{C}$. Total protein was quantified using a bicinchoninic acid assay kit (Beyotime Institute of Biotechnology). Equal amounts of protein were separated via 10-12\% SDS-PAGE and transferred electrophoretically to PVDF membranes (Invitrogen; Thermo Fisher Scientific, Inc.). The membranes were blocked with Tris buffer (Beyotime Institute of 
Table I. Sequences of primers used for reverse transcription-quantitative PCR.

\begin{tabular}{|c|c|c|}
\hline Gene & Sequence $\left(5^{\prime} \rightarrow 3^{\prime}\right)$ & Product size (bp) \\
\hline \multirow[t]{2}{*}{$\beta$-actin } & F: GCGTCCACCCGCGAGTACAA & 118 \\
\hline & R: ACATGCCGGAGCCGTTGTCG & \\
\hline \multirow[t]{2}{*}{ NDUFA1 } & F: TGCTGCCGGAAGAGCGGTGA & 189 \\
\hline & R: TCCTTGCCCCCGTTGGTGAACT & \\
\hline \multirow[t]{2}{*}{ NDUFA2 } & F: ACTGAGGACTGAACAAGCCCACCA & 223 \\
\hline & R: GCGACATCCCAGCGGGTAGC & \\
\hline \multirow[t]{2}{*}{ NDUFA13 } & F: CTACTGGAGAATAATGAGGTGGAAC & 175 \\
\hline & R: CCAGTTGGGCACATCTTTCA & \\
\hline \multirow[t]{2}{*}{ Mn-SOD } & F: GTGTCTGTGGGAGTCCAAGG & 149 \\
\hline & R: TGCTCCCACACATCAATCCC & \\
\hline \multirow[t]{2}{*}{ PGC-1 $1 \alpha$} & F: GGGGCACATCTGTTCTTCCA & 156 \\
\hline & R: GCTTGACTGGGATGACCGAA & \\
\hline \multirow[t]{2}{*}{ NRF1 } & F: ACACAGCATAGCCCATCTCG & 226 \\
\hline & R: GGTCATTTCACCGCCCTGTA & \\
\hline \multirow[t]{2}{*}{ NRF2 } & F: AGCAAGACTTGGGCCACTTA & 112 \\
\hline & R: TCTGGCTTCTTGCTCTTGGG & \\
\hline
\end{tabular}

NDUF, NADH: Ubiquinone oxidoreductase subunit; Mn-SOD, manganese superoxide dismutase; PGC-1 $\alpha$, peroxisome proliferator-activated receptor- $\gamma$ coactivator- $1 \alpha$; NRF, nuclear respiratory factor.

Biotechnology) containing 0.1\% Tween-20 (TBST) in 5\% milk for $1 \mathrm{~h}$ at room temperature and then incubated overnight with primary antibodies targeted against: NDUFA13 (17 kD;1:1,000; cat. no. ab110240; Abcam), AMPK (62 KD;1:1,000; cat. no. 5831; Cell Signaling Technology, Inc.), phosphorylated (p)-AMPK (62 KD;1:1,000; cat. no. 50081; Cell Signaling Technology, Inc.) and GAPDH (37 KD;1:10,000; cat. no. 5174; Cell Signaling Technology, Inc.). Following primary incubation, the membranes were incubated with a horseradish peroxidase-conjugated goat anti-rabbit secondary antibody (1:10,000; cat. no. HA1001; HuaBio Inc.) for $2 \mathrm{~h}$ at room temperature. Protein bands were visualized using an enhanced chemiluminescence system (EMD Millipore). ImageJ software (version 1.41; National Institutes of Health) was used to quantify the bands of each protein and GAPDH was used as the loading control.

Immunofluorescence assay. Cells were fixed with $4 \%$ paraformaldehyde for $30 \mathrm{~min}$ at room temperature and permeabilized with $0.1 \%$ Triton X-100 for $10 \mathrm{~min}$. Subsequently, cells were blocked with 3\% bovine serum albumin (Beyotime Institute of Biotechnology) for $2 \mathrm{~h}$ at room temperature and incubated with an anti-NDUFA13 primary antibody (1:50; cat. no. ab110240; Abcam) overnight at $4^{\circ} \mathrm{C}$. The slides were then washed with phosphate-buffered saline (PBS, Beyotime Institute of Biotechnology) and 0.1\% Tween-20 (PBST) and incubated with a Texas Red/Alexa fluor-conjugated secondary antibody for $1 \mathrm{~h}$ at room temperature. The slides were mounted using mounting medium, counterstained with 6-diamidino-2-phenylindole (Invitrogen; Thermo Fisher Scientific, Inc.) for $10 \mathrm{~min}$ at room temperature and observed using an IX71 microscope (Olympus Corporation; magnification, $\mathrm{x} 200$ ) and Image Pro Plus 3.0 software (Media Cybernetics, Inc.).
Reverse transcription-quantitative PCR (RT-qPCR). Total RNA was extracted using TRIzol ${ }^{\circledR}$ reagent (Thermo Fisher Scientific, Inc.). Total RNA was reverse transcribed into cDNA at $42^{\circ} \mathrm{C}$ for $1 \mathrm{~h}$ then $90^{\circ} \mathrm{C}$ for 5 min using a cDNA synthesis kit (Takara Biotechnology Co., Ltd.). Subsequently, qPCR was performed using SYBR green PCR Master Mix (Applied Biosystems; Thermo Fisher Scientific, Inc.). The following thermocycling conditions were used for the qPCR: Initial denaturation at $95^{\circ} \mathrm{C}$ for $2 \mathrm{~min}$; and 40 cycles of $95^{\circ} \mathrm{C}$ for $15 \mathrm{sec}$ and $60^{\circ} \mathrm{C}$ for $1 \mathrm{~min}$. The sequences of the primers used for qPCR are presented in Table I. mRNA expression levels were quantified using the $2^{-\Delta \Delta C q}$ method (19) and normalized to the internal reference gene $\beta$-actin.

Statistical analysis. Data are expressed as the mean \pm standard deviation of at least three independent experiments. Comparisons among multiple groups were analyzed using one-way ANOVA followed by Tukey's post hoc test. Statistical analyses were performed using GraphPad Prism software (version 7; GraphPad Software, Inc.). P $<0.05$ was considered to indicate a statistically significant difference.

\section{Results}

Metformin protects $H 9 C 2$ cells from $H G$-induced cytotoxicity. To investigate the effect of metformin on cardiomyocyte survival in various concentrations of glucose $(5.5-50 \mathrm{mM})$, H9C2 cell viability was assessed. Cell viability was significantly decreased following incubation with $33.3 \mathrm{mM}$ glucose (Fig. 1A) for at least $24 \mathrm{~h}$ (Fig. 1B) compared with the control group. Subsequently, cardiomyocytes were pretreated with metformin and then incubated with $33.3 \mathrm{mM}$ glucose for $36 \mathrm{~h}$. Metformin $(0.5$ and $1 \mathrm{mM})$ significantly increased 

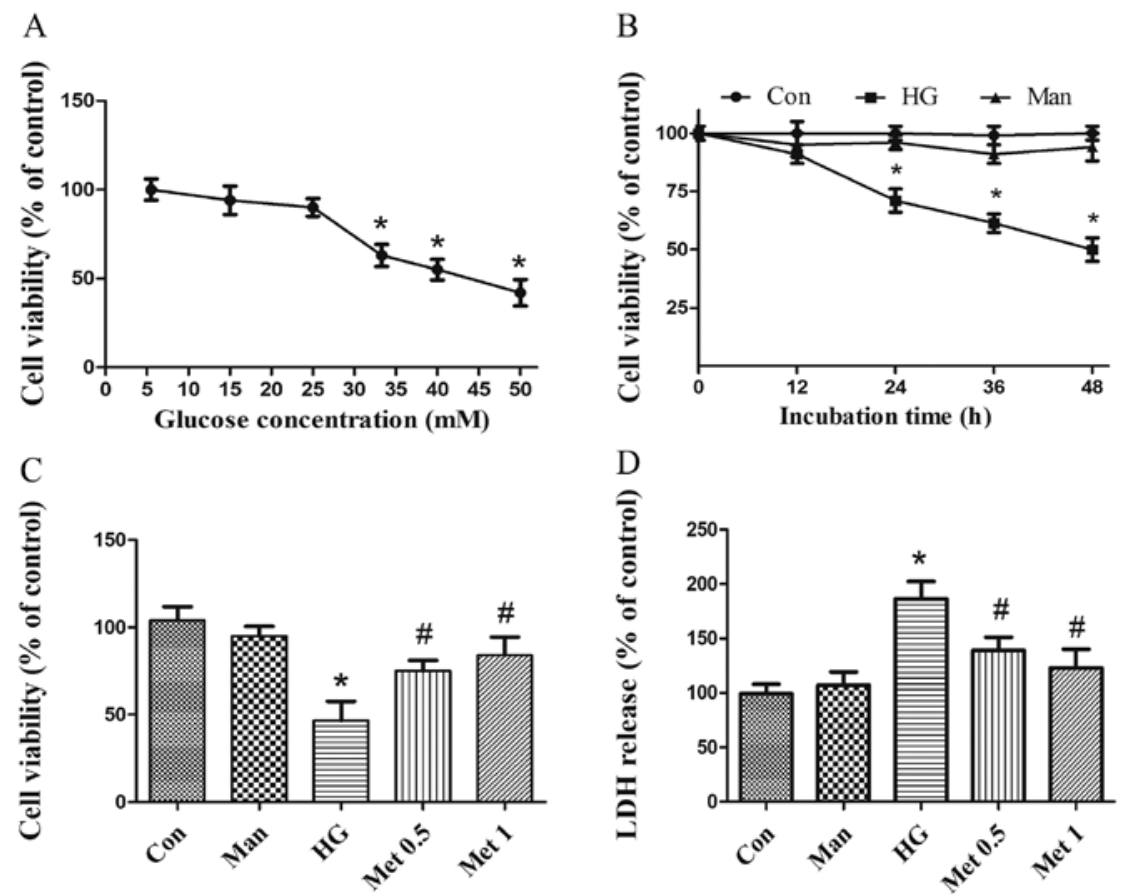

Figure 1. Effect of metformin on cardiomyocyte survival under high glucose conditions. (A) Effect of different concentrations of glucose on H9C2 cell viability. "P $<0.05$ vs. Con. (B) H9C2 cell viability following incubation with 5.5 glucose, $33.3 \mathrm{mM}$ glucose or $33.3 \mathrm{mM}$ Man for up to $48 \mathrm{~h}$. "P $<0.05$ vs. Con. (C) H9C2 cell viability following incubation with $33.3 \mathrm{mM}$ glucose and 0.5 or $1 \mathrm{mM}$ Met. ${ }^{*} \mathrm{P}<0.05$ vs. Con; ${ }^{\mathrm{P}}<0.05$ vs. HG. (D) H9C2 cell LDH release following incubation with $33.3 \mathrm{mM}$ glucose and 0.5 or $1 \mathrm{mM}$ Met. Man $(33.3 \mathrm{mM})$ was used as an osmotic control. ${ }^{\text {"P }}<0.05$ vs. Con; ${ }^{~} \mathrm{P}<0.05 \mathrm{vs}$. HG. LDH, lactate dehydrogenase; Con, control; HG, high glucose; Man, mannitol; Met, metformin.

A

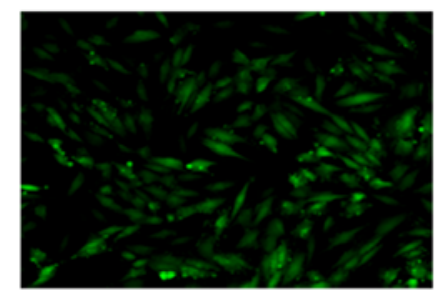

$\mathrm{B}$

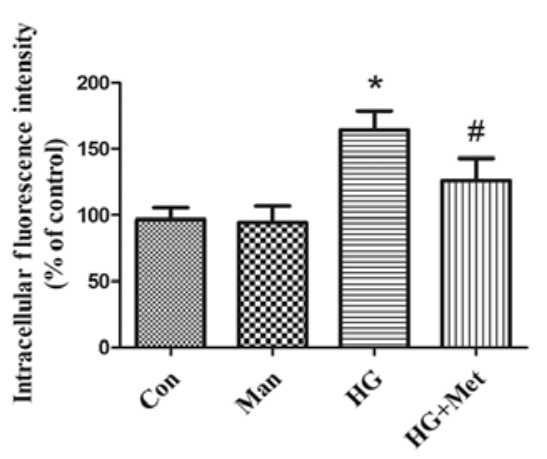

Man

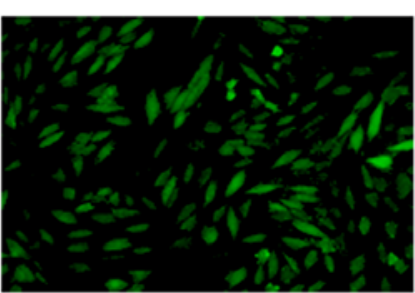

$\mathrm{C}$

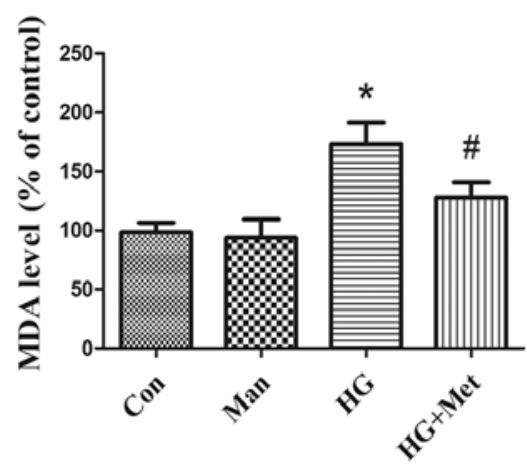

HG

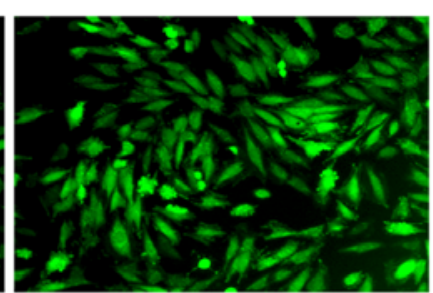

$\mathrm{D}$

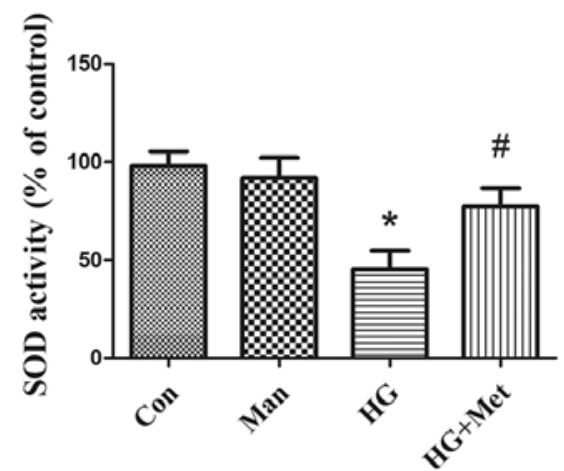

Figure 2. Effects of metformin (1 mM) on high glucose-induced oxidative stress in $\mathrm{H} 9 \mathrm{C} 2$ cells. Cells were divided into four groups: i) Con, normal medium; ii) Man, $33.3 \mathrm{mM}$ Man; iii) HG, $33.3 \mathrm{mM}$ glucose; and iv) HG + Met, $33.3 \mathrm{mM}$ glucose pretreated with $1 \mathrm{mM}$ Met. ROS levels were detected by (A) dichlorofluorescein fluorescence intensity and (B) quantified. Magnification, x200, Scale bars $=50 \mu \mathrm{m}$. (C) MDA and (D) SOD levels. " $\mathrm{P}<0.05$ vs. Con; " $\mathrm{P}<0.05$ vs. HG. Man, mannitol; HG, high glucose; Met, metformin; ROS, reactive oxygen species; MDA, malondialdehyde; SOD, superoxide dismutase; Con, control.

cell viability (Fig. 1C) and decreased LDH release (Fig. 1D) under HG conditions compared with the HG group. The results indicated that metformin rescued cardiomyocytes from HG-induced injury. Mannitol was used as an osmotic control and did not mimic the effects of $33.3 \mathrm{mM}$ glucose (Fig. 1). For subsequent experiments, $33.3 \mathrm{mM}$ glucose was used to induce a HG situation and $1 \mathrm{mM}$ metformin was applied, unless stated otherwise. 
A

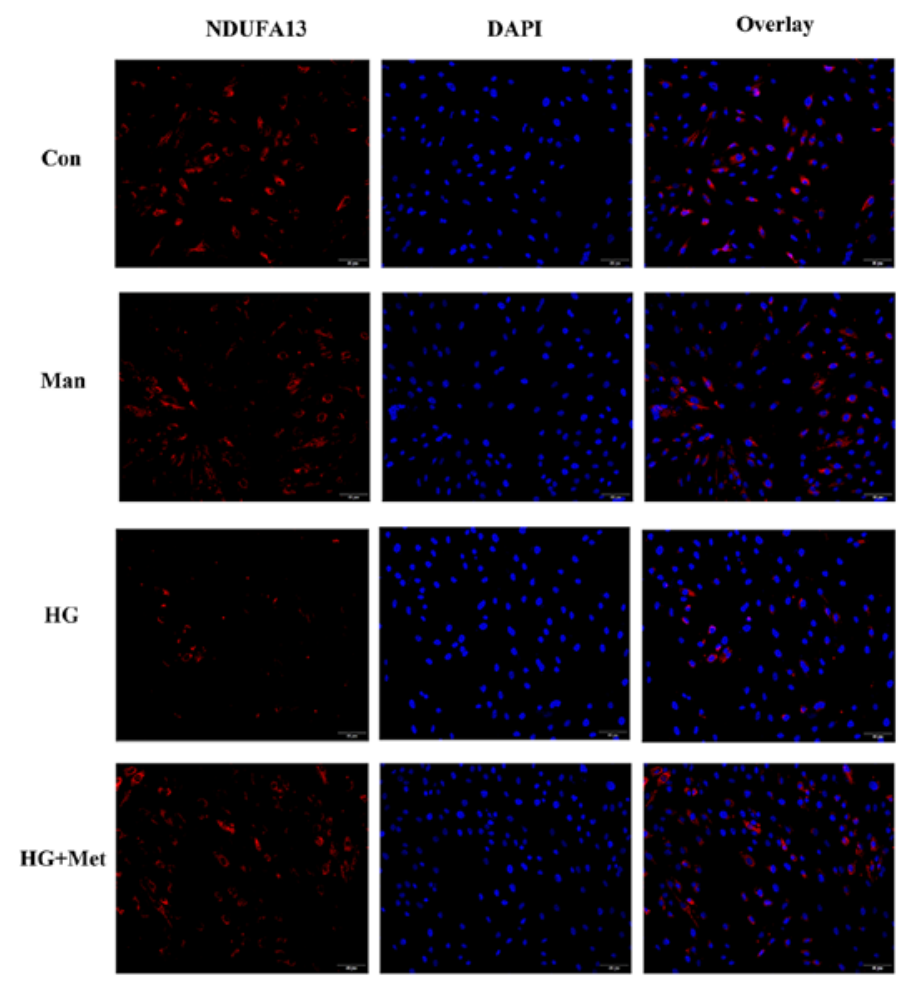

B
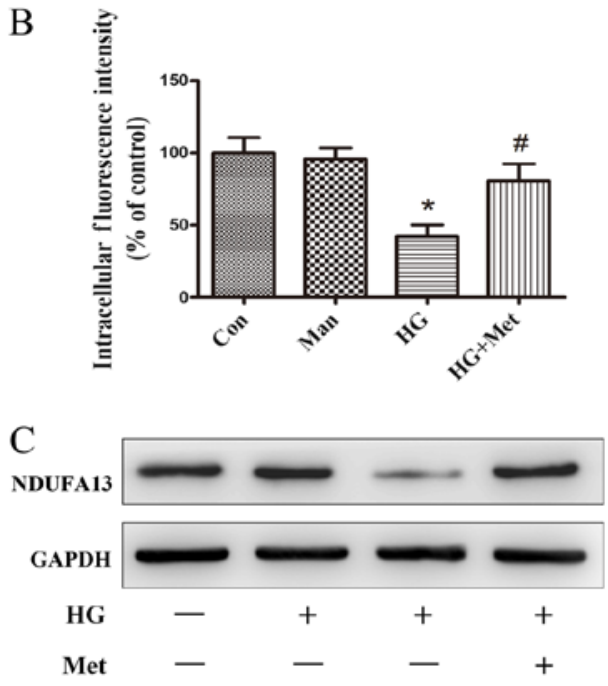

D

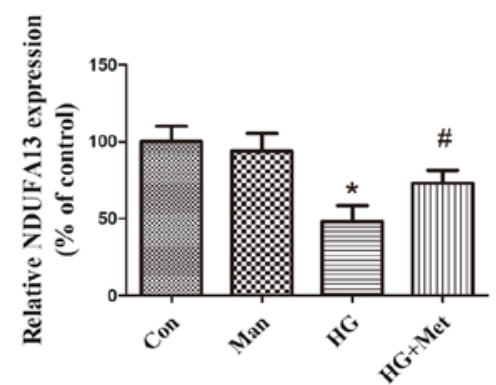

Figure 3. Effects of metformin (1 mM) on NDUFA13 expression in H9C2 cells under high glucose conditions. Cells were divided into four groups: i) Con, normal medium; ii) Man, $33.3 \mathrm{mM}$ Man; iii) HG, $33.3 \mathrm{mM}$ glucose; and iv) $\mathrm{HG}+$ Met, $33.3 \mathrm{mM}$ glucose pretreated with 1 mM Met. NDUFA13 protein expression was (A) evaluated by immunofluorescence and (B) quantified. Magnification, x200, Scale bars $=50 \mu \mathrm{m}$. (C) NDUFA13 protein expression levels were $(C)$ determined by western blotting and $(\mathrm{D})$ semi-quantified. ${ }^{*} \mathrm{P}<0.05$ vs. Con; ${ }^{\#} \mathrm{P}<0.05$ vs. HG. NDUFA13, NADH: Ubiquinone oxidoreductase subunit A13; Man, mannitol; HG, high glucose; Met, metformin; Con, control.

Clinically, the median plasma concentration of metformin is $330 \mu \mathrm{M}(20)$; however, the concentration of metformin is several times higher in tissues compared with in the blood (20). Therefore, intracellular metformin concentrations are $10-15 \%$ of the drug present in the medium (21), meaning the dose used in the present study was higher than the therapeutic dose.

Metformin suppresses $H G$-induced oxidative stress in $\mathrm{H} 9 \mathrm{C} 2$ cells. Markers of oxidative stress, including ROS, MDA and SOD, were detected. Compared with the control and mannitol groups, ROS and MDA levels were significantly increased in the high glucose group. However, metformin pretreatment significantly decreased ROS (Fig. 2A and B) and MDA (Fig. 2C) levels compared with the HG group. By contrast, HG significantly inhibited SOD activity compared with the control and mannitol groups, whereas metformin reversed HG-mediated inhibition of SOD activity in $\mathrm{H} 9 \mathrm{C} 2$ cells (Fig. 2D).

Metformin prevents $H G$-mediated downregulation of NDUFA13. To examine the effects of metformin on NDUFA13 expression, immunofluorescence and western blotting assays were performed. The immunofluorescence results suggested that the expression of NDUFA13 was significantly decreased in the HG group compared with the control and mannitol groups, but this effect was partly reversed by pretreatment with metformin (Fig. 3A and B). The western blotting results were consistent with the immunofluorescence results (Fig. 3C and D). Mannitol was used as the osmotic control and did not mimic the effects of $33.3 \mathrm{mM}$ glucose (Fig. 3).

\section{Metformin activates AMPK and induces mitochondrial} biogenesis. The activity of AMPK was evaluated by detecting the phosphorylation of Thr-172 (p-AMPK) via western blotting. p-AMPK expression levels were significantly decreased in the HG group compared with the control group, but metformin reversed HG-mediated effects (Fig. 4A and B).

RT-qPCR was performed to evaluate the effects of metformin on the mRNA expression levels of mitochondrial genes (NDUFA1, NDUFA2, NDUFA13 and Mn-SOD) and transcription factors (PGC-1 $\alpha$, NRF-1 and NRF-2). The results suggested that, compared with the control group, the expression levels of mitochondrial genes related to intracellular ROS production were significantly decreased in the HG group, which was reversed by metformin (Fig. 4C). Therefore, the results provided a potential explanation for the ability of metformin to reduce ROS production. Additionally, the inhibitory effect of $\mathrm{HG}$ on mitochondria biogenesis-related transcription factor expression was reversed by metformin pretreatment (Fig. 4D), which may be associated with metformin-mediated promotion of cardiomyocyte survival under high glucose conditions. 
A

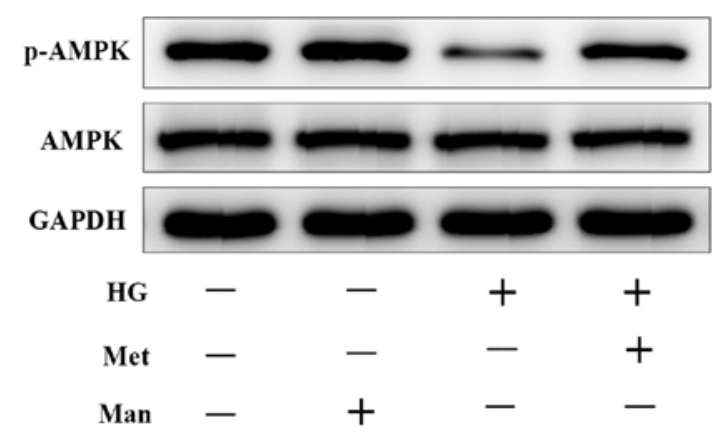

$\mathrm{C}$

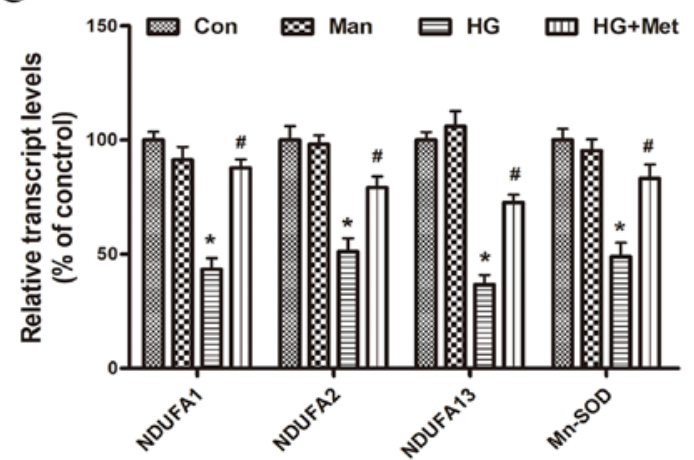

$\mathrm{B}$

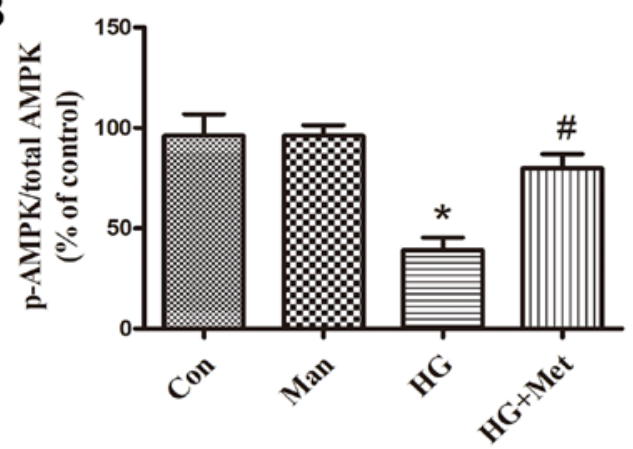

$\mathrm{D}$

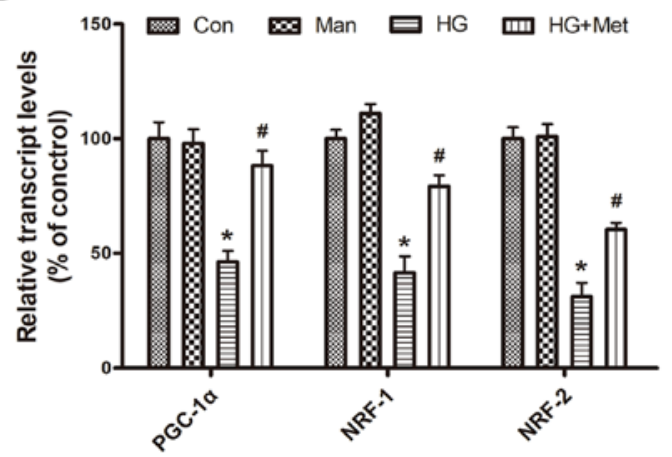

Figure 4. Effects of metformin ( $1 \mathrm{mM})$ on the expression of AMPK, p-AMPK, and mitochondrial biogenesis genes under high glucose conditions. Cells were divided into four groups: i) Con, normal medium; ii) Man, $33.3 \mathrm{mM}$ Man; iii) $\mathrm{HG}, 33.3 \mathrm{mM}$ glucose; and iv) $\mathrm{HG}+$ Met, $33.3 \mathrm{mM}$ glucose pretreated with $1 \mathrm{mM}$ Met. (A) Protein expression levels of p-AMPK and AMPK were (A) determined by western blotting and (B) the ratio of p-AMPK/AMPK was semi-quantified. mRNA expression levels of (C) NDUFA1, NDUFA2, NDUFA13, Mn-SOD, (D) PGC-1 $\alpha$, NRF1 and NRF2 were detected via reverse transcription-quantitative PCR. " $\mathrm{P}<0.05$ vs. Con; ${ }^{*} \mathrm{P}<0.05$ vs. HG. AMPK, AMP-activated protein kinase; $\mathrm{p}$, phosphorylated; Man, mannitol; HG, high glucose; Met, metformin; NDUF, NADH: Ubiquinone oxidoreductase subunit; Mn-SOD, manganese superoxide dismutase; PGC-1 $\alpha$, peroxisome proliferator-activated receptor- $\gamma$ coactivator- $1 \alpha$; NRF, nuclear respiratory factor; Con, control.

Mannitol was used as an osmotic control and did not mimic the effects of $33.3 \mathrm{mM}$ glucose (Fig. 4).

Compound $C$ reduced the expression of NDUFA13 and mitochondrial biogenesis via suppressing AMPK pathway. To examine the dependency of AMPK in metformin-induced mitochondrial biogenesis and NDUFA13 expression, a selective AMPK chemical inhibitor (Compound $\mathrm{C}$ ) was used. The results indicated that metformin-induced AMPK activation, as evidenced by an increase in p-AMPK expression levels, was blocked by pretreatment with Compound C (Fig. 5A and B). Compared with the HG + Met group, mitochondrial gene and mitochondrial biogenesis-related transcription factor expression levels were also significantly decreased in the $\mathrm{HG}+\mathrm{Met}+$ Comp C group (Fig. 5D and E). In addition, metformin-induced NDUFA13 upregulation reversed by Compound $\mathrm{C}$ under $\mathrm{HG}$ conditions (Fig. 5A and C). The results suggested that the roles of metformin in NDUFA13 expression and mitochondrial biogenesis were AMPK signaling pathway-dependent.

\section{Discussion}

In the present study, metformin exerted protective effects on H9C2 cardiomyocytes by suppressing HG-induced oxidative stress, as evidenced by ameliorating HG-induced decreases in cell viability and SOD levels, and increases in ROS and MDA levels. mRNA expression levels of the mitochondrial genes (NDUFA1, NDUFA2, NDUFA13 and Mn-SOD) were upregulated by metformin under HG conditions compared with the HG group. Furthermore, under HG conditions, the expression levels of the mitochondrial protein NDUFA13 were increased by metformin compared with the HG group. The results also indicated that mitochondrial biogenesis-related transcription factors (PGC-1 $\alpha, \mathrm{NRF} 1$ and NRF2) were targets of metformin. The present study identified the role of the AMPK signaling pathway in the mechanism underlying metformin-mediated regulation of NDUFA13 and mitochondrial biogenesis based on the effects of the AMPK inhibitor, Compound C. The results indicated that metformin protected cardiac cells against HG-induced oxidative stress via a mechanism involving p-AMPK, NDUFA13 and mitochondrial biogenesis.

Although the association between mitochondrial biogenesis and ROS production is not completely understood, it has been reported that hyperglycemia-induced ROS impairs mitochondrial biogenesis and mitochondrial function $(12,22)$. Metformin is an orally administered biguanide that is widely used to treat type 2 diabetes (4). The cardioprotective effects of metformin have received increasing attention and may be related to the ability of metformin to regulate oxidative stress; however, the exact mechanism is not completely understood (23). 
A

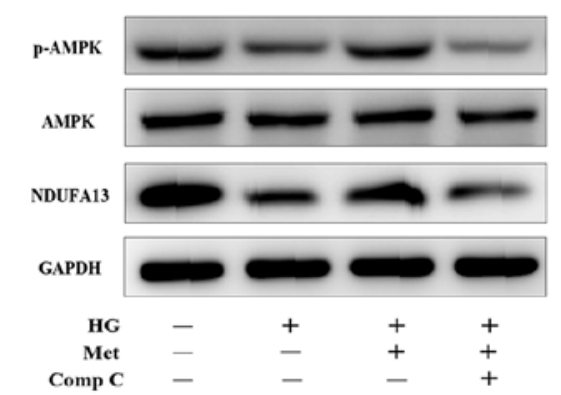

B

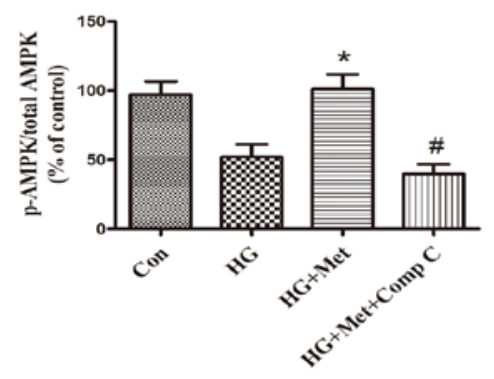

C

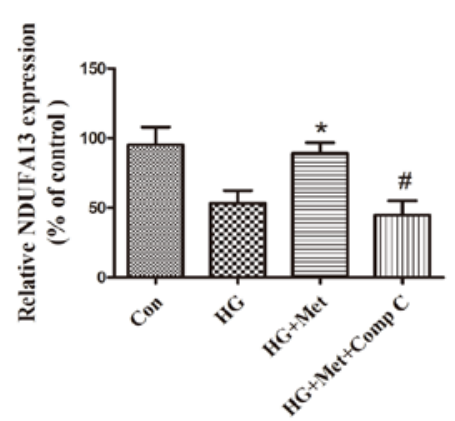

D

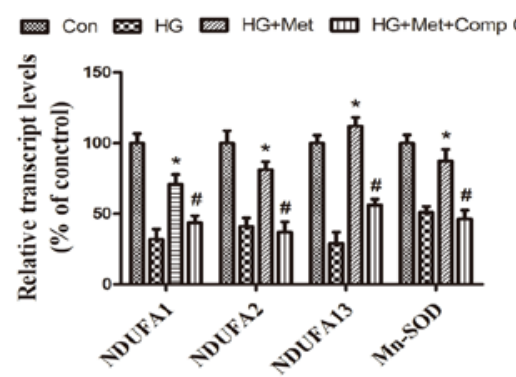

E

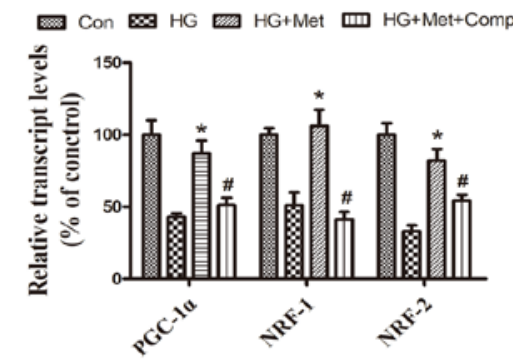

Figure 5. Role of the AMPK signaling pathway in the expression NDUFA13 and mitochondrial biogenesis. Cells were divided into four groups: i) Con, normal medium; ii) HG, $33.3 \mathrm{mM}$ glucose; iii) $\mathrm{HG}+$ Met, $33.3 \mathrm{mM}$ glucose pretreated with $1 \mathrm{mM}$ Met; and iv) $\mathrm{HG}+\mathrm{Met}+\mathrm{Comp}$ C, $33.3 \mathrm{mM}$ glucose pretreated with $1 \mathrm{mM}$ Met and Comp C. Protein expression levels were (A) determined by western blotting and (B) semi-quantified for the ratio of p-AMPK/AMPK and (C) NDUFA13. mRNA expression levels of (D) NDUFA1, NDUFA2, NDUFA13, Mn-SOD, (E) PGC-1 $\alpha$, NRF1 and NRF2 were measured via reverse transcription-quantitative PCR. ${ }^{*} \mathrm{P}<0.05$ vs. HG; ${ }^{\#} \mathrm{P}<0.05$ vs. HG + Met. AMPK, AMP-activated protein kinase; NDUF, NADH: Ubiquinone oxidoreductase subunit; HG, high glucose; Met, metformin; Comp C, Compound C; p, phosphorylated; Mn-SOD, manganese superoxide dismutase; PGC-1 $\alpha$, peroxisome proliferator-activated receptor- $\gamma$ coactivator- $1 \alpha$; NRF, nuclear respiratory factor; Con, control.

A previous study identified the role of metformin in mitochondrial biogenesis, which is an endogenous protective response to stress (8). Mitochondrial biogenesis is significantly impaired in cardiomyocytes from $\mathrm{db} / \mathrm{db}$ mice, as indicated by reduced mitochondrial DNA content and mitochondrial respiratory chain activity (24). Metformin administration promotes mitochondrial biogenesis and improves mitochondrial function in the skeletal muscle and human umbilical vein endothelial cells by activating PGC-1 $\alpha$ (25). Activated PGC- $1 \alpha$ is a powerful inducer of NRF-1, NRF-2 and mitochondrial transcription factor $\mathrm{A}$, which initiates the expression of nuclear and mitochondrial genes encoding mitochondrial respiratory chain proteins (26). Although the role of metformin in mitochondrial biogenesis has been investigated, the effects of metformin in cardiomyocytes have not been fully elucidated.

The results of the current study suggested that pretreatment with metformin increased the expression of mitochondrial complex I subcomplexes, including NDUFA1, NDUFA2 and NDUFA13, under HG conditions. Additionally, the expression levels of mitochondrial biogenesis-related transcription factors, such as PGC-1 $\alpha, \mathrm{NRF}-1$ and NRF-2, and the antioxidant enzyme, Mn-SOD, were regulated by metformin under HG conditions. The results suggested that metformin stimulated cardiomyocyte mitochondrial biogenesis, and may serve a critical role in cellular defense and cell survival responses to HG-induced oxidative stress.
The present study investigated the role of AMPK activation in metformin-mediated effects in cardiomyocytes. AMPK is a highly conserved sensor of cellular energy homeostasis and is the most recognized factor that mediates multiple effects of metformin (27). AMPK activation causes mitochondrial biogenesis (28). Mice expressing a dominant-negative form of AMPK in skeletal muscle are unable to increase mitochondrial biogenesis in response to energy deprivation (29). Activated AMPK stimulates PGC-1 $\alpha$, NRF1 and NRF-2, which in turn promote mitochondrial biogenesis (30). In the present study, metformin activated AMPK and enhanced the expression of PGC-1 $\alpha$, NRF1 and NRF-2 under HG conditions compared with the HG group. The expression of mitochondrial genes NDUFA1, NDUFA2, NDUFA13 and Mn-SOD was also regulated by metformin. The effects of metformin on expression were reversed by Compound $C$; therefore, the results suggested that AMPK served an important role in promoting mitochondrial biogenesis and the expression of mitochondrial genes in response to metformin. Additionally, alternative signaling pathways involving AMPK, Mn-SOD and PGC-1 $\alpha$, and those independent of mitochondrial genes should be investigated. Tumor suppressor p53-mediated PGC-1 $\alpha$ upregulation suppresses increased levels of oxidative stress via nuclear factor (erythroid-derived 2)-like2-mediated expression of Mn-SOD and $\gamma$-glutamyl cysteine ligase without modulating mitochondrial biogenesis (31). 
The present study suggested that the expression of the mitochondrial protein NDUFA13, a newly identified accessory subunit of mitochondrial complex I, was increased by metformin under HG conditions. Furthermore, the results indicated that NDUFA13 was regulated by metformin via an AMPK signal-dependent pathway, as evidenced by the inhibition of NDUFA13 induction in HG conditions by Compound $\mathrm{C}$.

NDUFA13 was originally identified as a death activator in tumor cells and was recognized as an indispensable subunit of mitochondrial complex I (32). Elimination of NDUFA13 prevents the assembly and electron transfer activity of complex I, and also influences other complexes in the mitochondrial respiratory chain (14). The role of NDUFA13 in H9C2 cardiomyocytes and the relationship among NDUFA13, metformin and AMPK are not completely understood. However, mutations in the genes encoding subunits of complex I and complex I increase ROS generation (33). NDUFA13 downregulation increases basal ROS generation, which may serve as a survival signal by activating the STAT3/Bcl-2 signaling pathway (34). In the present study, NDUFA13 expression levels were decreased in HG-induced H9C2 cardiomyocytes compared with control H9C2 cardiomyocytes. Therefore, it was hypothesized that reduced expression levels of NDUFA13 in $\mathrm{H} 9 \mathrm{C} 2$ cardiomyocytes were responsible for elevated ROS production under HG conditions, which may explain the antioxidant effects of metformin.

Moreover, it was observed that mitochondrial Mn-SOD was upregulated by metformin under HG conditions. Mn-SOD is a major antioxidant mitochondrial enzyme that serves as a primary ROS regulator. Mn-SOD-deficient mitochondria are more susceptible to oxidative stress and exhibit ultrastructural damage (35). The present study demonstrated that increased Mn-SOD expression might be involved in the mechanism underlying metformin-mediated amelioration of oxidative stress.

The present study had several limitations. AMPK kinase inhibitor Compound $\mathrm{C}$ can also inhibit vascular endothelial growth factor and bone morphogenetic proteins receptors $(36,37)$. Therefore, further investigations into the effects of AMPK deficiency, for example, are required to verify the results of the present study. In addition, only the $\mathrm{H} 9 \mathrm{c} 2$ cell line was used in the present study; therefore, in vivo studies or in vitro studies involving additional cell lines are required to verify the conclusions of the present study.

In conclusion, the present study suggested that metformin protected cardiomyocytes against HG-induced oxidative stress. The results indicated that metformin-mediated protection occurred at least in part via promoting mitochondrial biogenesis and NDUFA13 expression. In addition, the present study indicated that the AMPK signaling pathway was associated with the mechanisms underlying metformin-mediated regulation of NDUFA13 and mitochondrial biogenesis. The results suggested that AMPK may serve as a potential therapeutic target for normalizing mitochondrial function in diabetes, and NDUFA13 may serve as a useful target for designing novel pharmacological approaches to prevent diabetic complications.

\section{Acknowledgements}

Not applicable.

\section{Funding}

The present study was supported by the Natural Science Foundation of China (grant no. 81771496), Natural Science Foundation of China (grant no. 881800331), School of Medicine, Shanghai Jiao Tong University (grant no. 16XJ21006), Shanghai Municipal Health Commission (grant no. 201940079) and Interdisciplinary Program of Shanghai Jiao Tong (grant no. YG2017QN23).

\section{Availability of data and materials}

The datasets used and/or analyzed during the current study are available from the corresponding author on reasonable request.

\section{Authors' contributions}

QYZ, LLW and XDL designed the study. YGL, GYW, YZ, MLY and YGB performed the experiments. XDL and YGL drafted the manuscript. MW and XBL helped with the statistical analysis. QYZ, MW and XBL revised the paper. All authors reviewed the manuscript and approved the final manuscript.

\section{Ethics approval and consent to participate}

Not applicable.

\section{Patient consent for publication}

Not applicable.

\section{Competing interests}

The authors declare that they have no competing interests.

\section{References}

1. Hayat SA, Patel B, Khattar RS and Malik RA: Diabetic cardiomyopathy: Mechanisms, diagnosis and treatment. Clin Sci (Lond) 107: 539-557, 2004.

2. Cai L and Kang YJ: Oxidative stress and diabetic cardiomyopathy: A brief review. Cardiovasc Toxicol 1: 181-193, 2001.

3. Khullar M, Al-Shudiefat AA, Ludke A, Binepal G and Singal PK: Oxidative stress: A key contributor to diabetic cardiomyopathy. Can J Physiol Pharmacol 88: 233-240, 2010.

4. Sanchez-rangel E and Inzucchi SE: Metformin: Clinical use in type 2 diabetes. Diabetologia 60: 1586-1593, 2017.

5. Intensive blood-glucose control with sulphonylureas or insulin compared with conventional treatment and risk of complications in patients with type 2 diabetes (UKPDS 33). UK prospective diabetes study (UKPDS) group. Lancet 352: 837-853, 1998.

6. Varjabedian L, Bourji M, Pourafkari L and Nader ND: Cardioprotection by metformin: Beneficial effects beyond glucose reduction. Am J Cardiovasc Drugs 18: 181-193, 2018.

7. Calvert JW, Gundewar S, Jha S, Greer JJM, Bestermann WH, Tian R and Lefer DJ: Acute metformin therapy confers cardioprotection against myocardial infarction via AMPK-eNOS-mediated signaling. Diabetes 57: 696-705, 2008.

8. Hu M, Ye P, Liao H, Chen M and Yang F: Metformin protects H9C2 cardiomyocytes from high-glucose and hypoxia/reoxygenation injury via inhibition of reactive oxygen species generation and inflammatory responses: Role of AMPK and JNK. J Diabetes Res 2016: 2961954, 2016.

9. Kim AS, Miller EJ and Young LH: AMP-activated protein kinase: A core signalling pathway in the heart. Acta Physiol (Oxf) 196: 37-53, 2009. 
10. Paiva MA, Rutter-Locher Z, Gonçalves LM, Providência LA, Davidson SM, Yellon DM and Mocanu MM: Enhancing AMPK activation during ischemia protects the diabetic heart against reperfusion injury. Am J Physiol Heart Circ Physiol 300: $\mathrm{H} 2123-\mathrm{H} 2134,2011$.

11. Kusmic C, L'abbate A, Sambuceti G, Drummond G, Barsanti C, Matteucci M, Cao J, Piccolomini F, Cheng J and Abraham NG: Improved myocardial perfusion in chronic diabetic mice by the up-regulation of pLKB1 and AMPK signaling. J Cell Biochem 109: 1033-1044, 2010.

12. Zheng A, Li H, Xu J, Cao K, Li H, Pu W, Yang Z, Peng Y, Long J, Liu J and Feng Z: Hydroxytyrosol improves mitochondrial function and reduces oxidative stress in the brain of $\mathrm{db} / \mathrm{db}$ mice: Role of AMP-activated protein kinase activation. Br J Nutr 113 1667-1676, 2015.

13. Vakifahmetoglu-norberg H, Ouchida AT and Norberg E: The role of mitochondria in metabolism and cell death. Biochem Biophys Res Commun 482: 426-431, 2017.

14. Huang G, Lu H, Hao A, Ng DC, Ponniah S, Guo K, Lufei C, Zeng Q and Cao X: GRIM-19, a cell death regulatory protein, is essential for assembly and function of mitochondrial complex I. Mol Cell Biol 24: 8447-8456, 2004.

15. Li YG, Han BB, Li F, Yu JW, Dong ZF, Niu GM, Qing YW, Li JB, Wei M and Zhu W: High glucose induces down-regulated GRIM-19 expression to activate STAT3 signaling and promote cell proliferation in cell culture. PLoS One 11: e0153659, 2016.

16. Sazanov LA: Respiratory complex I: Mechanistic and structural insights provided by the crystal structure of the hydrophilic domain. Biochemistry 46: 2275-2288, 2007.

17. Zhang Y, Liu X, Zhang L, Li X, Zhou Z, Jiao L, Shao Y, Li M, Leng B, Zhou Y, et al: Metformin protects against $\mathrm{H} 2 \mathrm{O} 2$-induced cardiomyocyte injury by inhibiting the miR-1a-3p/GRP94 pathway. Mol Ther Nucleic Acids 13: 189-197, 2018.

18. Chang J, Jung HH, Yang JY, Lee S, Choi J, Im GJ and Chae SW: Protective effect of metformin against cisplatin-induced ototoxicity in an auditory cell line. J Assoc Res Otolaryngol 15: $149-158,2014$

19. Livak KJ and Schmittgen TD: Analysis of relative gene expression data using real-time quantitative PCR and the 2(-Delta Delta C(T)) method. Method 408: 402-408, 2001

20. Foretz M, Guigas B, Bertrand L, Pollak M and Viollet B Metformin: From mechanisms of action to therapies. Cell Metab 20: 953-966, 2014

21. Dowling RJO, Lam S, Bassi C, Mouaaz S, Aman A, Kiyota T, Al-Awar R, Goodwin PJ and Stambolic V: Metformin pharmacokinetics in mouse tumors: Implications for human therapy. Cell Metab 23: 567-568, 2016

22. Xie L, Zhu X,Hu Y,Li T, Gao Y, Shi Y and Tang S: Mitochondrial DNA oxidative damage triggering mitochondrial dysfunction and apoptosis in high glucose-induced HRECs. Invest Ophthalmol Vis Sci 49: 4203-4209, 2008

23. Kukidome D, Nishikawa T, Sonoda K, Imoto K, Fujisawa K, Yano M, Motoshima H, Taguchi T, Matsumura T and Araki E: Activation of AMP-activated protein kinase reduces hyperglycemia-induced mitochondrial reactive oxygen species production and promotes mitochondrial biogenesis in human umbilical vein endothelial cells. Diabetes 55: 120-127, 2006.

24. Yan W, Zhang H, Liu P, Wang H, Liu J, Gao C, Liu Y, Lian K, Yang L, Sun L, et al: Impaired mitochondrial biogenesis due to dysfunctional adiponectin-AMPK-PGC-1 $\alpha$ signaling contributing to increased vulnerability in diabetic heart. Basic Res Cardiol 108: 329, 2013.
25. Karnewar S, Neeli PK, Panuganti D, Kotagiri S, Mallappa S, Jain N, Jerald MK and Kotamraju S: Metformin regulates mitochondrial biogenesis and senescence through AMPK mediated H3K79 methylation: Relevance in age-associated vascular dysfunction. Biochim Biophys Acta Mol Basis Dis 1864: 1115-1128, 2018.

26. Chen SD, Yang DI, Lin TK, Shaw FZ, Liou CW and Chuang YC: Roles of oxidative stress, apoptosis, PGC- $1 \alpha$ and mitochondrial biogenesis in cerebral ischemia. Int J Mol Sci 12: 7199-7215, 2011.

27. Adeghate $\mathrm{E}$ and Singh J: Structural changes in the myocardium during diabetes-induced cardiomyopathy. Heart Fail Rev 19: $15-23,2014$

28. Nanjaiah H and Vallikannan B: Enhanced phosphorylation of AMPK by lutein and oxidised lutein that lead to mitochondrial biogenesis in hyperglycemic HepG2 cells. J Cell Biochem 120: 15255-15267, 2019.

29. Zong H, Ren JM, Young LH, Pypaert M, Mu J, Birnbaum MJ and Shulman GI: AMP kinase is required for mitochondrial biogenesis in skeletal muscle in response to chronic energy deprivation. Proc Natl Acad Sci USA 99: 15983-15987, 2002.

30. Song P, Kwon Y, Yea K, Moon HY, Yoon JH, Ghim J, Hyun H, Kim D, Koh A, Berggren PO, et al: Apolipoprotein al increases mitochondrial biogenesis through AMP-activated protein kinase. Cell Signal 27: 1873-1881, 2015.

31. Aquilano K, Baldelli S, Pagliei B, Cannata SM, Rotilio G and Ciriolo MR: p53 orchestrates the PGC-1 $\alpha$-mediated antioxidant response upon mild redox and metabolic imbalance. Antioxid Redox Signal 18: 386-399, 2013.

32. Moreira S, Correia M, Soares P and Máximo V: GRIM-19 function in cancer development. Mitochondrion 11: 693-699, 2011.

33. Fato R, Bergamini C, Leoni S, Strocchi P and Lenaz G: Generation of reactive oxygen species by mitochondrial complex I: Implications in neurodegeneration. Neurochem Res 33: 2487-2501, 2008.

34. Hu H, Nan J, Sun Y, Zhu D, Xiao C, Wang Y,Zhu L, Wu Y, Zhao J, Wu R, et al: Electron leak from NDUFA13 within mitochondrial complex I attenuates ischemia-reperfusion injury via dimerized STAT3. Proc Natl Acad Sci USA 114: 11908-11913, 2017.

35. Williams MD, Van Remmen H, Conrad CC, Huang TT, Epstein CJ and Richardson A: Increased oxidative damage is correlated to altered mitochondrial function in heterozygous manganese superoxide dismutase knockout mice. J Biol Chem 273: 28510-28515, 1998.

36. Yu PB, Hong CC, Sachidanandan C, Babitt JL, Deng DY, Hoyng SA, Lin HY, Bloch KD and Peterson RT: Dorsomorphin inhibits BMP signals required for embryogenesis and iron metabolism. Nat Chem Biol 4: 33-41, 2008.

37. Hao J, Ho JN, Lewis JA, Karim KA, Daniels RN, Gentry PR, Hopkins CR, Lindsley CW and Hong CC: In vivo structure-activity relationship study of dorsomorphin analogues identifies selective VEGF and BMP inhibitors. ACS Chem Biol 5: 245-253, 2010.

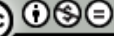

This work is licensed under a Creative Commons Attribution-NonCommercial-NoDerivatives 4.0 International (CC BY-NC-ND 4.0) License. 